\title{
Role of aspartate ammonia-lyase in Pasteurella multocida
}

\author{
Zui Wang ${ }^{1}$, Li Li ${ }^{1}$, Peng Liu ${ }^{1,2}$, Chen Wang ${ }^{1,2}$, Qin Lu', Lina Liu', Xiaozhong Wang ${ }^{2}$, Qingping Luo ${ }^{1,3^{*}}$ and \\ Huabin Shao ${ }^{1,3^{*}}$ (D)
}

\begin{abstract}
Background: Pasteurella multocida is responsible for a highly infectious and contagious disease in birds, leading to heavy economic losses in the chicken industry. However, the pathogenesis of this disease is poorly understood. We recently identified an aspartate ammonia-lyase (aspA) in P. multocida that was significantly upregulated under ironrestricted conditions, the protein of which could effectively protect chicken flocks against $P$. multocida. However, the functions of this gene remain unclear. In the present study, we constructed aspA mutant strain $\triangle$ aspA:kan and complementary strain $C \triangle a s p A:: k a n$ to investigate the function of aspA in detail.

Result: Deletion of the aspA gene in P. multocida resulted in a significant reduction in bacterial growth in LB (LuriaBertani) and $\mathrm{MH}$ (Mueller-Hinton) media, which was rescued by supplementation with $20 \mathrm{mM}$ fumarate. The mutant strain $\triangle$ aspA::kan showed significantly growth defects in anaerobic conditions and acid medium, compared with the wild-type strain. Moreover, growth of $\triangle a s p A:$ :kan was more seriously impaired than that of the wild-type strain under iron-restricted conditions, and this growth recovered after supplementation with iron ions. AspA transcription was negatively regulated by iron conditions, as demonstrated by quantitative reverse transcription-polymerase chain reaction. Although competitive index assay showed the wild-type strain outcompetes the aspA mutant strain and $\triangle a s p A:: k a n$ was significantly more efficient at producing biofilms than the wild-type strain, there was no significant difference in virulence between the mutant and the wild-type strains.
\end{abstract}

Conclusion: These results demonstrate that aspA is required for bacterial growth in complex medium, and under anaerobic, acid, and iron-limited conditions.

Keywords: Pasteurella multocida, Aspartate ammonia-lyase, Iron acquisition, Virulence

\section{Background}

Pasteurella multocida is a capsulated, Gram-negative facultative anaerobic bacterium responsible for fowl cholera in poultry, leading to great economic losses in commercial layer flocks and local chicken breeds [1]. P. multocida is currently classified into five serogroups (A, B, D, E, and F) based on its capsular antigens, and fowl cholera is mainly caused by strains of serovars A, F, and very rarely D [2]. Once a

\footnotetext{
*Correspondence: qingping0523@163.com; shhb1961@163.com 'Institute of Animal Husbandry and Veterinary Sciences, Hubei Academy of Agricultural Sciences, Special one, Nanhuyaoyuan, Hongshan District, Wuhan 430064, China

Full list of author information is available at the end of the article
}

chicken flock becomes infected with the bacterium, it may become endemic and difficult to remove, resulting in repeated infectious episodes [3]. However, the molecular basis of $P$. multocida pathogenesis is still poorly understood.

Aspartate ammonia-lyase (aspA) has been identified in various other Gram-negative bacteria, including Salmonella enterica [4], Actinobacillus pleuropneumoniae [5] and Escherichia coli [6]. This enzyme is involved in catalyzing the reversible conversion of L-aspartate to form fumarate and release ammonia [7], and plays a vital role in the production of L-aspartate [8]. The addition of L-aspartate significantly increased the survival of wild-type, but not asp $A$ mutant, $Y$. pseudotuberculosis in minimum essential

(c) The Author(s). 2020 Open Access This article is licensed under a Creative Commons Attribution 4.0 International License, which permits use, sharing, adaptation, distribution and reproduction in any medium or format, as long as you give appropriate credit to the original author(s) and the source, provide a link to the Creative Commons licence, and indicate if changes were made. The images or other third party material in this article are included in the article's Creative Commons licence, unless indicated otherwise in a credit line to the material. If material is not included in the article's Creative Commons licence and your intended use is not permitted by statutory regulation or exceeds the permitted use, you will need to obtain permission directly from the copyright holder. To view a copy of this licence, visit http://creativecommons.org/licenses/by/4.0/ The Creative Commons Public Domain Dedication waiver (http://creativecommons.org/publicdomain/zero/1.0/) applies to the data made available in this article, unless otherwise stated in a credit line to the data. 
medium at $\mathrm{pH} 4.5$ [9]. In addition to its role in an acidsurvival system, aspA is also important for anaerobic respiration, and fumarate produced by the decomposition of L-aspartate can serve as a terminal electron acceptor under anaerobic conditions [10]. The asp $A$ gene was significantly upregulated under iron-restricted conditions in various bacteria including C. jejuni [11], A. pleuropneumoniae [12], Edwardsiella ictaluri [13], and P. multocida [14], suggesting that asp $A$ might be related to iron acquisition. Meanwhile, an iron-restricted environment often triggers the expression of virulence factors in pathogens [15], indicating the need to determine if aspA is an important virulence factor in $P$. multocida.

In the present study, we constructed asp $A$ mutant strain $\triangle a s p A:: k a n$ and complementary strain $\mathrm{C}^{\triangle} a s p A:: k a n$ to investigate the role of aspA in the growth of $P$. multocida in complex medium, and under anaerobic, acid, and ironlimited conditions, and in P. multocida infection in vivo.

\section{Results}

Identification of $P$. multocida $\triangle$ aspA::kan mutant

The whole aspA gene (1419 bp) was replaced with a 902 bp kanamycin-resistance cassette using allelic exchange through a recombinant suicide vector. The mutant strain $\triangle a s p A:: k a n$ was confirmed by PCR screening using primers T1/2, aspA1/2, and Kan1/2 (Fig. 1a and Table 2). Primers T1/2 were both designed outside the homology arms of aspA. The amplicon size of the deleted alleles was $517 \mathrm{bp}$ less than the wild-type. Primers aspA1/2 were designed to target the whole aspA gene. The amplification product was present in the parent strain but not in the asp $A$ mutant. In addition, primers Kan $1 / 2$ were designed to target the whole Kan cassette, which was only present in the aspA mutant (Fig. 1b). To further characterize the aspA mutant, the expressions of aspA were detected in the parent strain $\mathrm{C}_{48-1}$ and complementary strain $C^{\triangle} a s p A:: k a n$ but not in the mutant strain $\triangle a s p A:: k a n$ or control strain $\triangle a s p A:: k a n$ (pAL99S) (Fig. $1 c$ ), indicating that the aspA gene was successfully deleted from $\mathrm{C} 48-1$.

\section{AspA is essential for growth of $P$. multocida}

We compared the growth rates of C48-1, $\triangle a s p A:: k a n$, $\mathrm{C} \triangle a s p A:: k a n$ and $\triangle a s p A:: k a n$ (pAL99S) by measuring their growth curves in TSB, MH, and LB media under aerobic conditions. The four strains showed similar

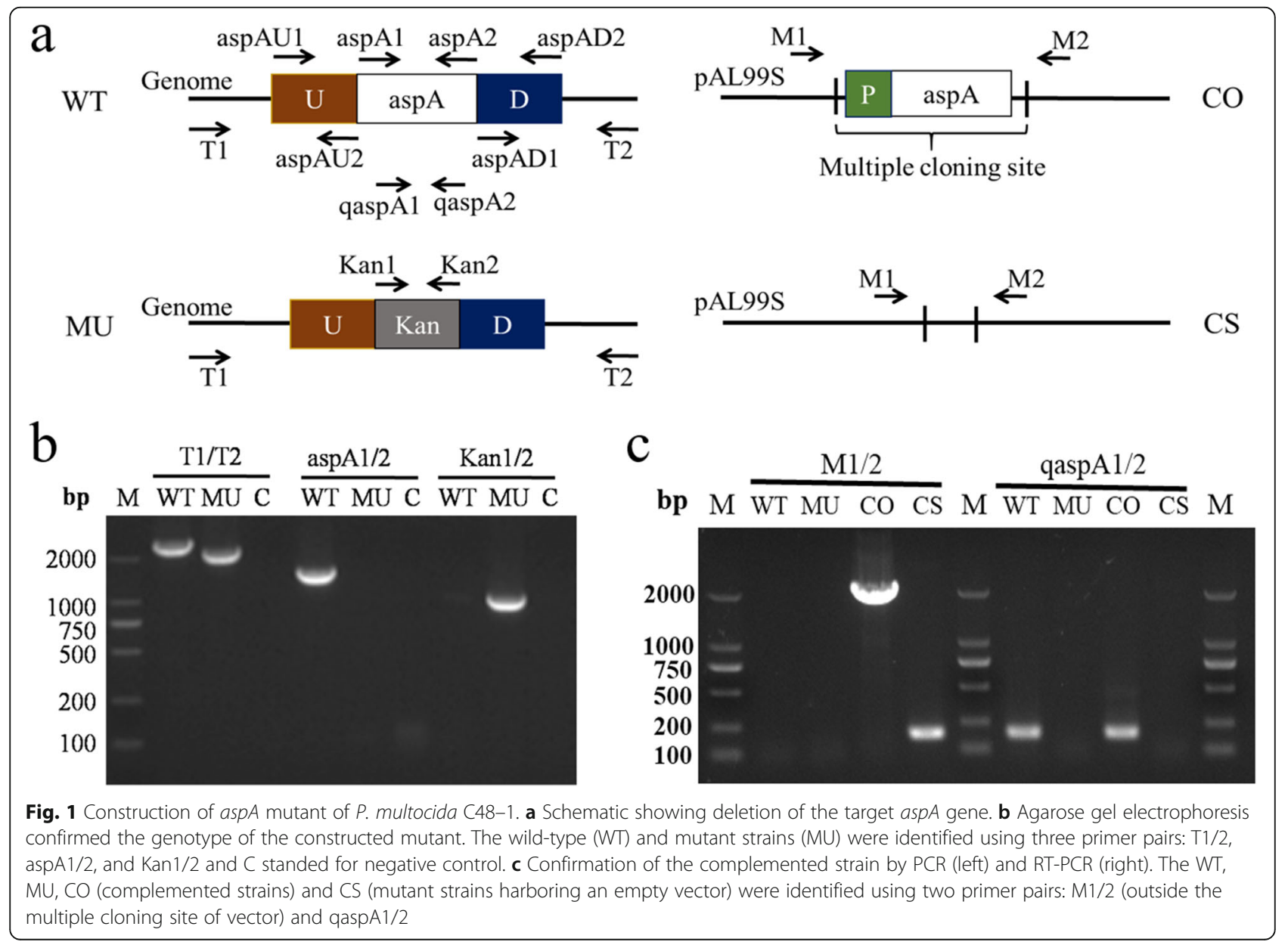


growth in TSB medium, but $\triangle a s p A:: k a n$ and $\triangle a s p A::$ kan (pAL99S) growth were relatively inhibited in LB and $\mathrm{MH}$ medium while the growth of complementation strain $\mathrm{C} a \operatorname{asp} A:: k a n$ was restored (Fig. 2a, b, c). Loss of aspA delayed the time to entry into logarithmic phase and significantly reduced the maximum growth. The final $\mathrm{OD}_{600}$ values of the asp $A$ mutant were 0.44 in $\mathrm{LB}(P<0.01)$ and 0.23 in $\mathrm{MH}(P<0.01)$, compared with 1.30 and 0.82 , respectively, for the wild-type strain. Interestingly, the growth defects of the mutant were partly recovered by supplementation of LB and $\mathrm{MH}$ with $20 \mathrm{mM}$ fumarate prior to inoculation (Fig. $2 \mathrm{~d}, \mathrm{e}$ ). The final $\mathrm{OD}_{600}$ values increased from 0.45 to 0.85 in $\mathrm{LB}(P<0.01)$ and from 0.24 to 0.52 in $\mathrm{MH}(P<0.01)$. These results indicated that fumarate, as the product of amino acid catabolism through $\operatorname{asp} A$, was important for the growth of $P$. multocida.

\section{AspA is required for $P$. multocida growth under anaerobic} conditions

To determine if aspA affected the growth of P. multocida under anaerobic conditions, we compared the abilities of the parent, mutant, complementary and control strains to grow under anaerobic conditions in TSB. Growth of all four strains was inhibited under anaerobic conditions, but growth of the mutant and control strains was slower than that of the wild-type and complementary strain (Fig. 3). The final $\mathrm{OD}_{600}$ of $\triangle a s p A:: k a n$ was about 0.28 , compared with 0.78 for the wild-type strain $(P<0.01)$. As expected, the growth defects of the mutant were partly recovered by supplementation with $20 \mathrm{mM}$ fumarate prior to inoculation (Fig. 3). The final $\mathrm{OD}_{600}$ values increased from 0.28 to 0.59 under anaerobic conditions $(P<0.01)$. These results suggest that loss of aspA could lead to growth defects under anaerobic conditions and fumarate might play a vital role for $P$. multocida growth under anaerobic conditions.

\section{AspA is related to acid survival in $P$. multocida}

To determine if aspA is involved in the acid survival of P. multocida, we compared the abilities of the parent, mutant, complementary and control strains to grow under acid conditions in TSB in presence of oxygen. After $1 \mathrm{~h}$ of incubation, the densities of four strains in TSB $(\mathrm{pH}=7.3)$ were approximately $6.83 \times 10^{6} \mathrm{CFU} / \mathrm{mL}$ (Fig. 4). However, the density of $\mathrm{C} 48-1$ colonies was $3.92 \times 10^{5} \mathrm{CFU} / \mathrm{mL}$ in acid medium (TSB, $\mathrm{pH}=5$ ), which was 12.58 -fold higher than $\triangle a s p A:: k a n(P<0.01)$. At the same time, the density of $C^{\triangle} a s p A:: k a n$ colonies was

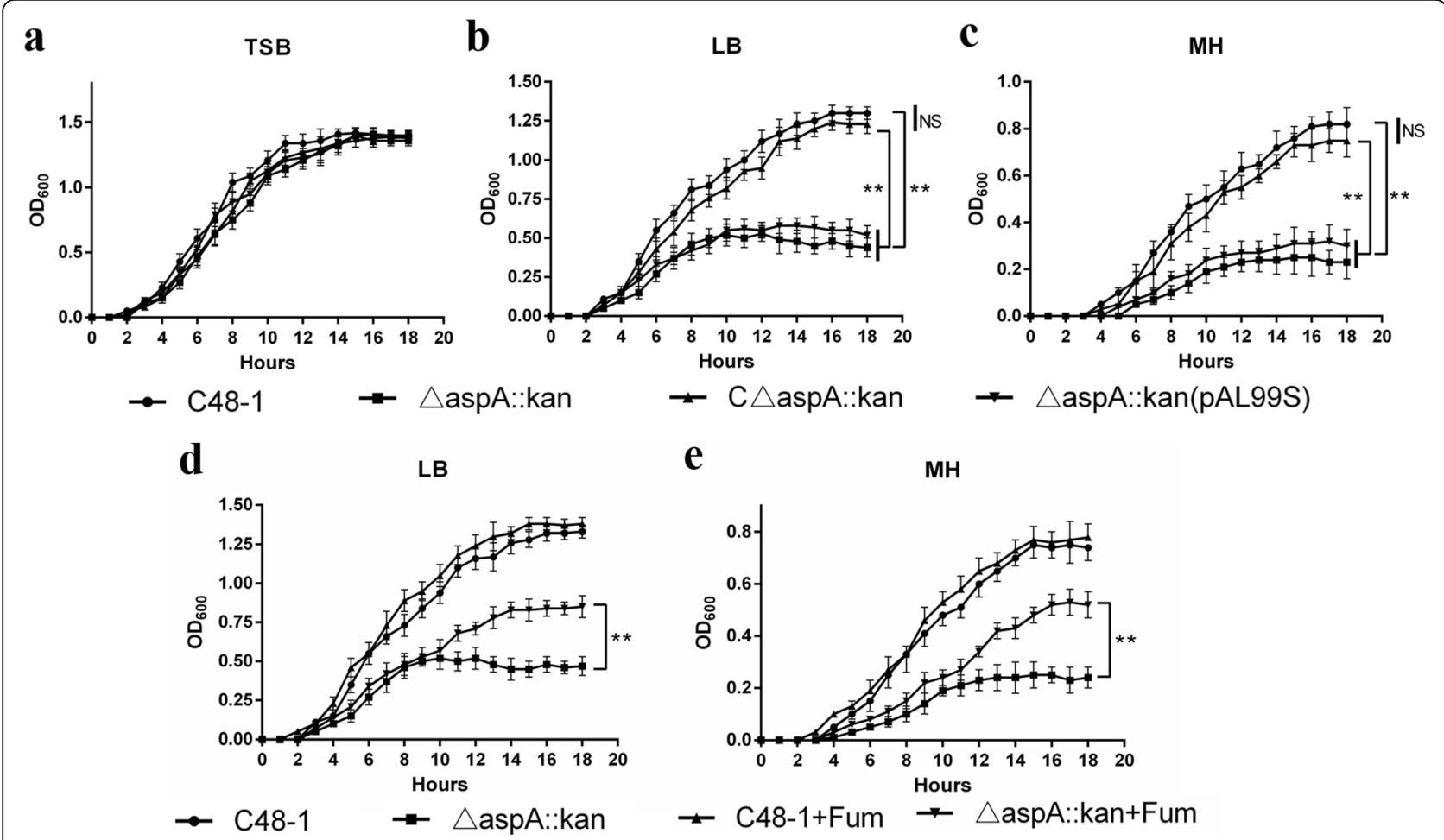

Fig. 2 Growth of C48-1, $\triangle$ aspA:kan, C $\triangle$ aspA::kan and $\triangle$ aspA::kan (pAL99S) in complex media under aerobic conditions. The growth curves were measured in (a) TSB, (b) LB and (c) MH. Growth of C48-1 and $\triangle$ aspA::kan in LB (d) and MH (e) supplemented with fumarate. The growth curves of C48-1 and $\triangle a s p A:$ :kan were determined after incubation in LB and $\mathrm{MH}$ with or without $20 \mathrm{mM}$ fumarate at $37^{\circ} \mathrm{Cfor} 18 \mathrm{~h}$. Data analyzed by Student's t-test. ${ }^{*} P<0.05 ;{ }^{*} P<0.01$ and NS (non-significant) for $P>0.05$ 


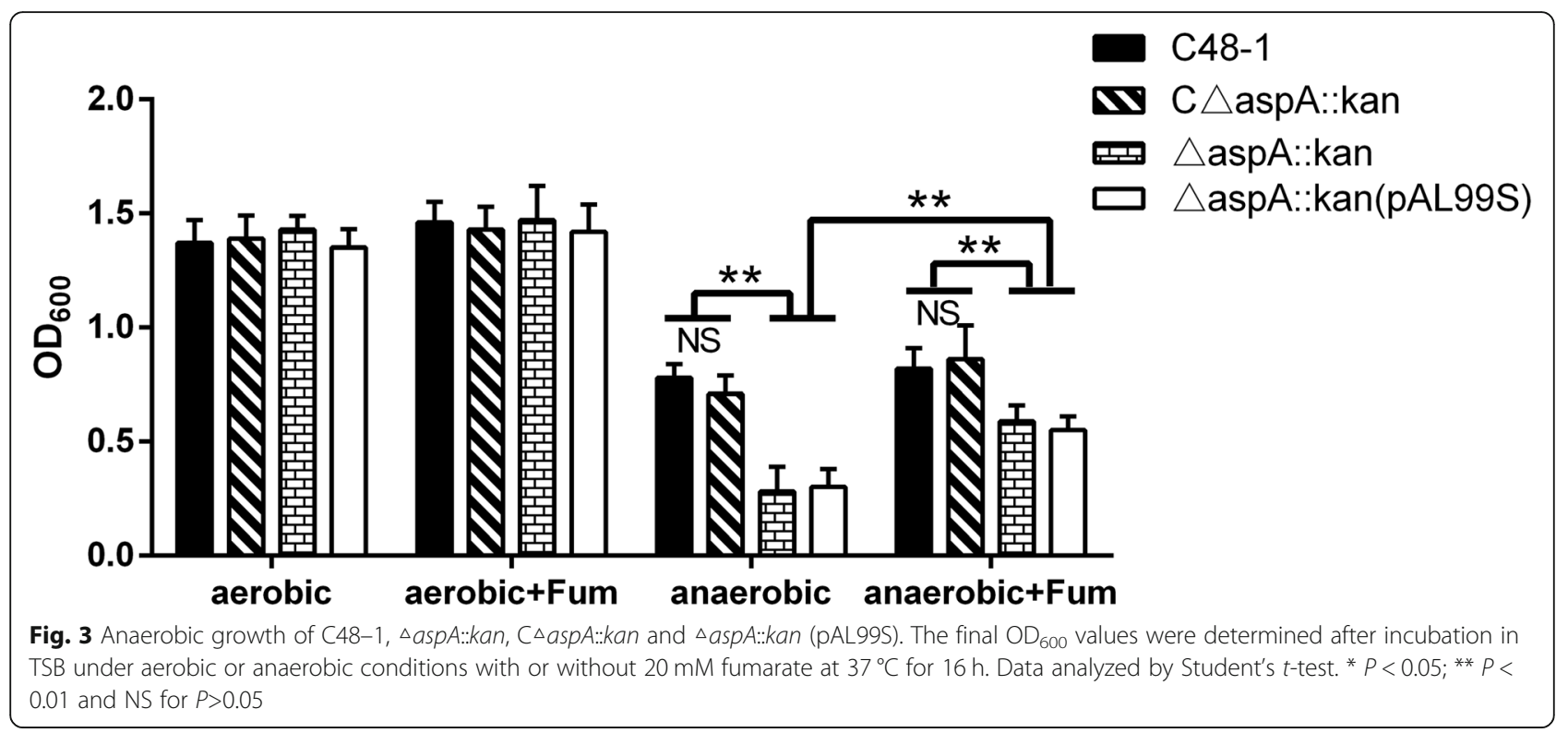

almost the same as $\mathrm{C} 48-1$. This indicated that loss of aspA reduced the acid resistance of $P$. multocida.

\section{AspA is related to iron acquisition in P. multocida}

We examined the role of asp $A$ in iron acquisition in $P$. multocida. The effect of iron depletion was determined by measuring the end-point OD of cultures in TSB containing the iron chelator 2,2'-dipyridyl (DPD) at concentrations of $0-300 \mu \mathrm{M}$ under aerobic conditions. The DPD sensitivities of the wild-type and mutant strains were compared (Fig. 5a). The growth of both strains was inhibited in TSB supplemented with 150 and $200 \mu \mathrm{M}$ DPD, and growth was almost absent at 250 and $300 \mu \mathrm{M}$
DPD. The growth of the mutant was significantly slower than that of the wild-type strain in the presence of $150 \mu \mathrm{M}$ DPD, with a reduction in $\mathrm{OD}_{600}$ from 1.02 to $0.6(P<0.01)$. We therefore chose $150 \mu \mathrm{M}$ DPD as the optimal working concentration to create a growth curve. Loss of aspA delayed the time of entry into the logarithmic phase and significantly reduced the maximum growth $(P<0.01$, Fig. $5 \mathrm{~b})$ while the growth of complementation strain $C^{\triangle} a s p A:: k a n$ was restored. These results suggested that the mutant strain was more sensitive to the iron-depleted environment, and that aspA was involved in iron acquisition in P. multocida. In addition, we also determined the ability of the mutant

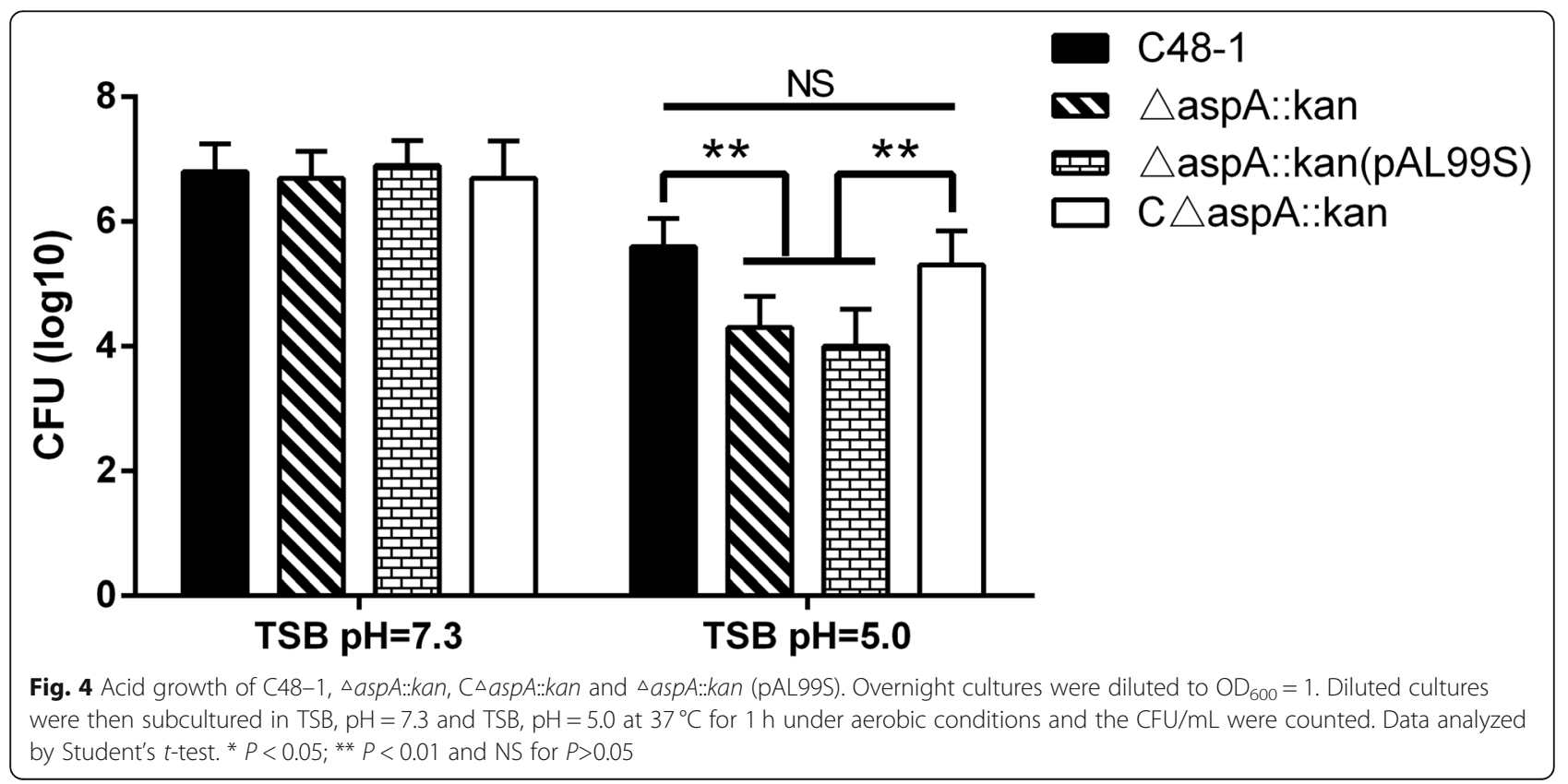




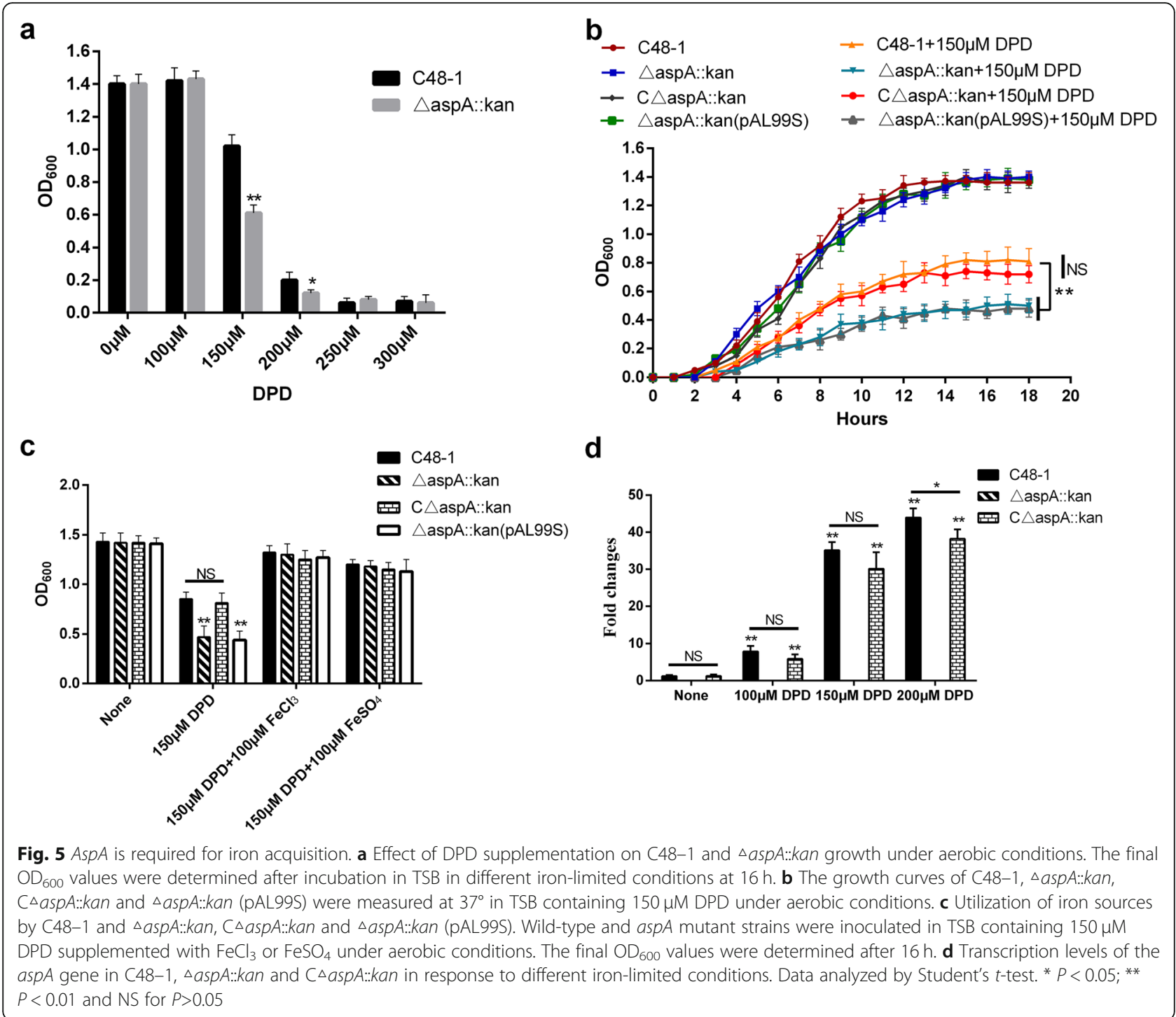

strain to utilize different iron sources in iron-depleted medium. Growth of $\triangle a s p A:: k a n$ and wild-type C48-1 was inhibited in TSB containing $150 \mu \mathrm{M}$ DPD, but growth of both was restored by addition of $100 \mathrm{mM}$ $\mathrm{FeCl}_{3}$ or $\mathrm{FeSO}_{4}$, with no significant difference between the mutant and wild-type strains (Fig. 5c). We therefore concluded that loss of aspA affected the absorption of chelated iron rather than free iron ions. To clarify if $\operatorname{asp} A$ was negatively regulated by iron ions, we determined the relative mRNA expression levels of aspA in C48-1, $\triangle$ aspA::kan, $\mathrm{C} \triangle a s p A:: k a n$ under various ironlimited conditions. The mRNA expressions of aspA were significantly upregulated under iron-limited conditions in $C 48-1$ and $C \triangle a s p A:: k a n(P<0.01$, Fig. 5 d). asp $A$ expression increased with increasing iron chelator. These results demonstrated that aspA was negatively regulated by the iron concentration in $P$. multocida, indicating that $\operatorname{asp} A$ plays an important role in chelated iron acquisition in P. multocida.

\section{Effect of aspA deletion on biofilm formation}

We explored the effect of aspA on biofilm formation in $P$. multocida by crystal violet staining. The mutant strain was significantly more efficient at producing biofilms than the wild-type strain $C 48-1 \quad(P<0.01$, Fig. 6). In addition, $\mathrm{C} 48-1$ produced more biofilms in iron-limited than that in iron-repleted conditions $(P<0.01$, Fig. 6), while biofilm production by the mutant was unaffected.

\section{Virulence of the aspA mutant strain}

We examined the role of aspA in virulence in 55-dayold healthy chickens infected with the mutant and wildtype strains, respectively. At a challenge dose of $10 \mathrm{CFU}$, survival rates were the same between the mutant and 


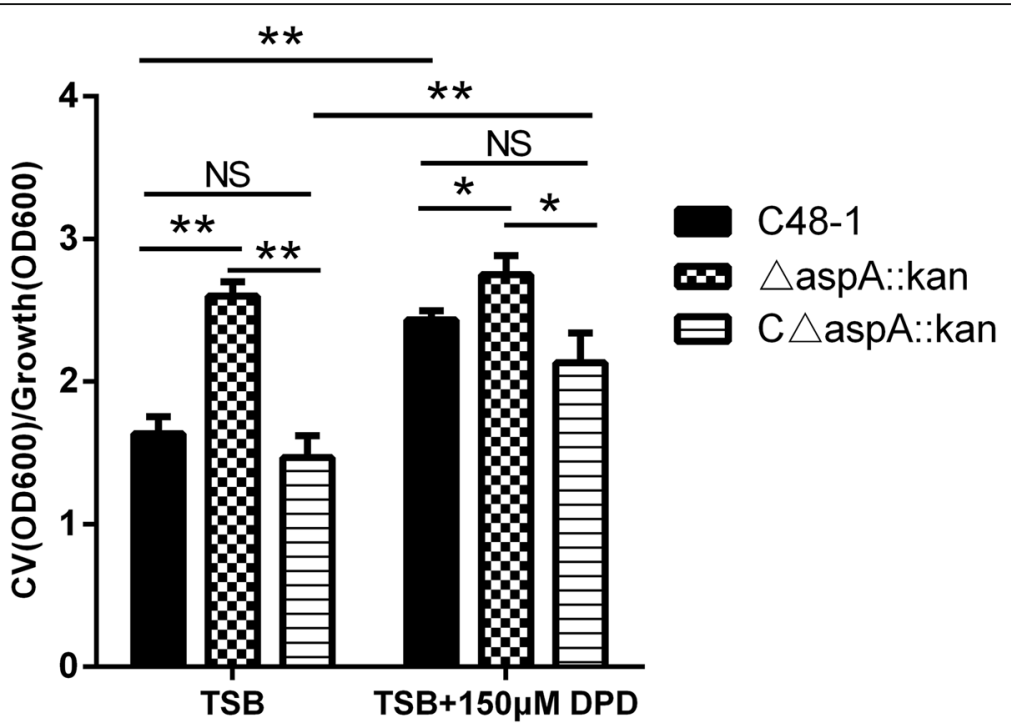

Fig. 6 Biofilm formation assessed by crystal violet staining. Biofilms were grown in TSB with or without $150 \mu M$ DPD at $37^{\circ} \mathrm{C}$ for $48 \mathrm{~h}$ in six-well plates under aerobic conditions. Biofilm biomass was measured by crystal violet staining and expressed relative to the final culture density. Data analyzed by Student's $t$-test. ${ }^{*} P<0.05 ;{ }^{*} P<0.01$ and NS for $P>0.05$

wild groups (7/10, 70\%) (Fig. 7). Moreover, the survival of the wild-type and mutant groups with a challenge dose of $100 \mathrm{CFU}$ were 20 and $30 \%$, respectively. These results indicated that the asp $A$ gene could not be related to the virulence of $P$. multocida in chickens.

We further explored the role of aspA in the colonization dynamics of $P$. multocida during systemic infection by competitive infection of 55-day-old healthy chickens with a 1:1 ratio of $C 48-1$ and the aspA mutant. The bacterial loads of the asp $A$ mutant strain in the spleen (209-fold reduction, $P<0.01)$ and liver $(115$-fold reduction, $P<0.01$ ) at $24 \mathrm{~h}$ post-infection were significantly reduced compared with the parent strain C48-1 (Fig. 8a). The aspA mutant was also significantly outcompeted by the wild-type strain in the spleen (263-fold reduction, $P<0.01$ ) and liver (182fold reduction, $P<0.01$ ) at $72 \mathrm{~h}$ post-infection (Fig. $8 \mathrm{c}$ ). At the same time, the colonization abilities were mostly restored in the complementary strain (Fig. 8b, d). These results showed that the wild-type strain outcompetes the aspA mutant strain during the infection.

\section{Discussion}

We previously identified an aspartate ammonia-lyase $(\operatorname{asp} A)$ that was significantly upregulated under ironrestricted conditions and the protein of which could effectively protect chicken flocks against $P$. multocida [14]. This suggested that aspA might play an important role in the survival of these bacteria under iron-restricted conditions and could be related to the virulence of $P$. multocida. We therefore further studied the function of the aspA gene by constructing asp $A$ mutant strain $\triangle a s p A:: k a n$ and complementary strain $\mathrm{C}^{\triangle} a s p A:: k a n$, and assessed its functions in growth in complex medium and under anaerobic, acid, and iron-limited conditions, and during infection in vivo.

AspA is involved in catalyzing the reversible conversion of L-aspartate to form fumarate and release ammonia [7], which is essential for the production of L-aspartic acid as an important carbon source for various bacteria [16]. Loss of aspA might thus affect the utilization of aspartic acid and inhibit the growth of bacteria. In the present study, we examined the effect of aspA mutation on the ability of $P$. multocida to grow in different complex media. We demonstrated that $\triangle a s p A:: k a n$ had dramatic growth defects in LB and $\mathrm{MH}$ media compared with the wild-type strain, though this was rescued by supplementation with $20 \mathrm{mM}$ fumarate. Similar results were found in $C$. jejuni. The effect of the aspA mutation on the depletion of amino acids in $\mathrm{MH}$ media after $48 \mathrm{~h}$ growth was determined in C. jejuni. Only serine was significantly utilized by this mutant while proline, aspartate and glutamate couldn't be used in comparison to the WT [17]. Fumarate, as the product of aspartate through aspA, could restore partially of the growth defect [17]. This suggests that amino acid catabolism involving asp $A$ was crucial for the growth of $P$. multocida in complex media. In addition, fumarate was not only indispensable for bacterial growth, but also served as a terminal electron acceptor under anaerobic conditions [18]. Fumarate is produced via the malate dehydrogenase and aspA pathways, respectively [19]. Transcriptome analysis showed that malate dehydrogenase was downregulated under anaerobic conditions [20] whereas aspA was increased [21], suggesting that aspA produced fumarate rather than malate dehydrogenase under anaerobic conditions. In the present 


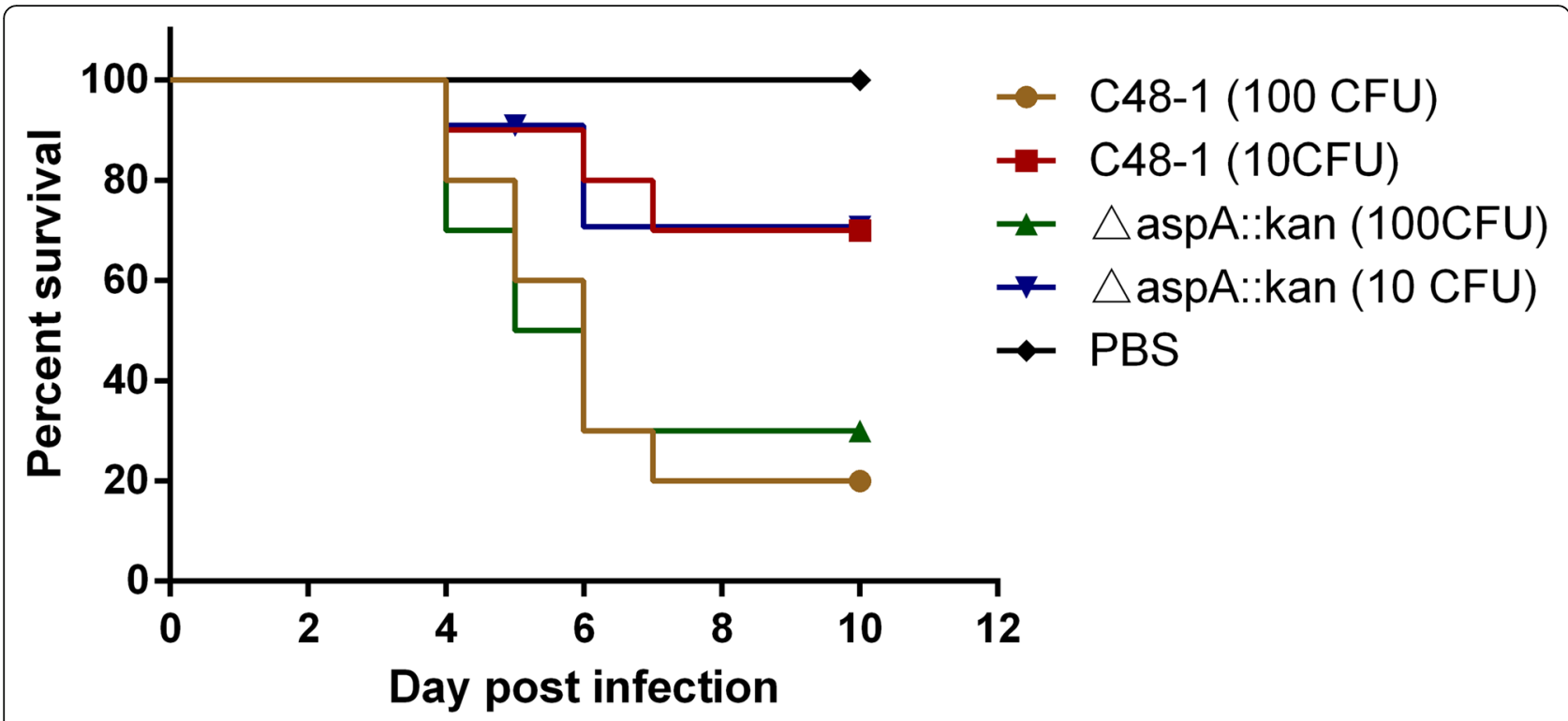

Fig. 7 Animal challenge experiment. Healthy chickens (55 days old) were injected intramuscularly with 10 or 100 CFU of parental C48-1 or mutant $\triangle$ aspA::kan strain and mortality was recorded daily for 12 days after challenge
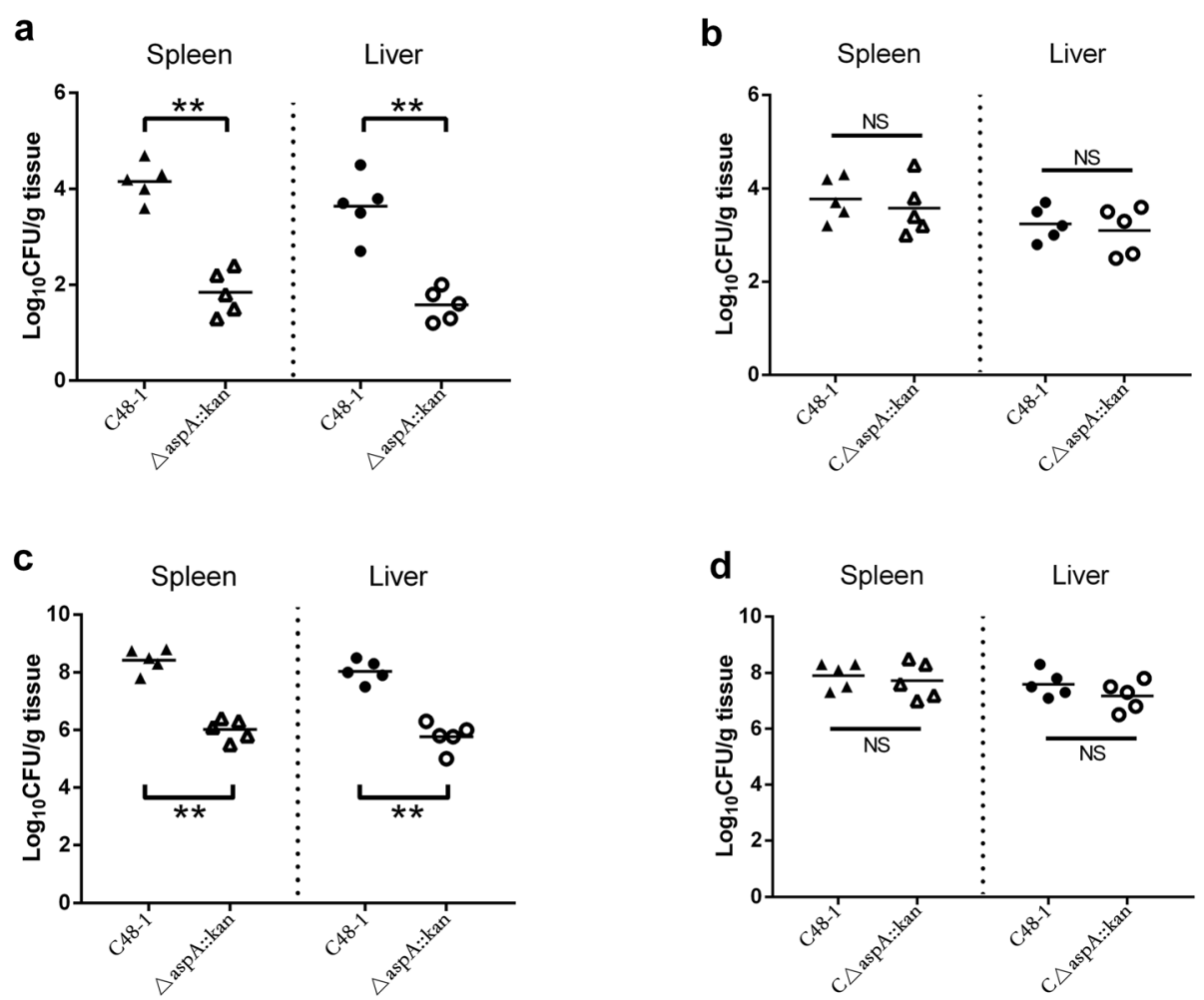

Fig. 8 Competitive infection by the wildtype C48-1 with mutant strains $\triangle a s p A: k a n$ or complemented strains C $\triangle$ aspA::kan in vivo. C48-1 and $\triangle$ aspA::kan or C $\triangle$ aspA::kan were mixed at a 1:1 ratio and $200 \mu$ of the mixture containing 100 CFU of each strain was inoculated into 55 -day-old healthy chickens. Bacteria were isolated from the spleens and livers at $24 \mathrm{~h} \mathrm{(a),} \mathrm{(b)} \mathrm{and} 72 \mathrm{~h}$ (c), (d) post-infection. Data points represent the CFU/ $\mathrm{g}$ of individual animals in the organs indicated; bars show median values $(n=5)$. Data analyzed by Student's $t$-test. ${ }^{*} P<0.05$; ${ }^{* *} P<0.01$ and NS for $P>0.05$ 
study, the $\operatorname{asp} A$ mutant strain $\triangle a s p A:: k a n$ showed significantly decreased growth under anaerobic conditions over a $16 \mathrm{~h}$ incubation period compared with the wild-type strain and this growth recovered after supplementation $20 \mathrm{mM}$ fumarate, suggesting that asp $A$ was important for anaerobic growth in vitro. Ammonia is also a product of amino acid catabolism through aspA, and may be responsible for increasing the intracellular $\mathrm{pH}$ [9]. A novel aspartatedependent acid-survival system involving asp $A$ has been identified in $Y$. pseudotuberculosis [9]. The present study demonstrated the existence of a similar aspartate acidsurvival system in P. multocida. The survival of C48-1 was 12.58-fold higher than that of $\triangle a s p A:: k a n$ after $1 \mathrm{~h}$ of incubation in acid medium.

Iron is indispensable for bacterial growth and iron acquisition is an important aspect of the pathogenesis of many bacteria [22]. Various iron-uptake systems have been found to play an essential role in virulence in $P$. multocida, particularly in different animal host environments [23]. The major objective of this study was to explore the role of aspA in iron utilization and the pathogenesis in $P$. multocida.

Amino acid homology analysis suggested that the structural and evolutionary relationships of $\operatorname{asp} A$ were closely related to fumarase (fumC) [24], which is known to be related to iron acquisition in many bacteria [25-28]. As their common product, fumarate could act as an electron acceptor during iron-uptake [29]. These results suggested that asp $A$ might also play a role in iron acquisition in $P$. multocida. In the present study, the mutant strain $\triangle a s p A:: k a n$ was more sensitive to iron-limited conditions and showed impaired growth in TSB containing $150 \mu \mathrm{M}$ DPD compared with the wild-type strain, thus supporting a role for asp $A$ in ironuptake. We also demonstrated that the addition of different iron ions could improve the growth of $\triangle a s p A:: k a n$, and concluded that loss of asp $A$ affected the absorption of chelated rather than free iron. Iron-uptake related genes are usually modulated by iron concentration, while only a handful of genes are not [22]. We monitored the transcription of $\operatorname{asp} A$ under different iron-limited conditions and found that aspA was negatively regulated by iron levels in P. multocida. Moreover, iron is not only involved in the regulation of iron-uptake genes, but also in the formation of biofilms [30], though the promotion or inhibition of biofilm formation depends on the species of bacteria [31-34]. In this study, either iron-limited conditions or loss of asp $A$ promoted the formation of biofilms by $P$. multocida. Although several iron-uptake systems are involved in biofilm formation $[35,36]$, the mechanism by which iron signaling regulates biofilm formation is unclear. The current results revealed that $\operatorname{asp} A$ is likely to play an important role in iron acquisition in $P$. multocida.

For most bacterial pathogens, the ability to acquire iron from the host is directly related to their virulence
[37]. Knocking out iron-associated proteins can thus reduce the virulence of various bacteria, including $P$. multocida $[23,38]$. Although there was no previous evidence relating $\operatorname{asp} A$ to iron acquisition, the virulence of the asp $A$ mutant has been explored in other bacteria. Pigs challenged with aspA mutants of $A$. pleuropneumoniae showed lower lung lesion scores than those challenged with the parent controls [5]. Ability of aspA mutant $C$. jejuni to persist in the intestines of chickens was impaired relative to the wild-type strain [17]. However, although a competitive index assay showed the wild-type strain outcompetes the $\operatorname{asp} A$ mutant strain, there was no significant difference in the virulence of $P$. multocida between the $\operatorname{asp} A$ mutant and the wild strains in this study. The possible reasons are as follows. On the one hand, the virulence of $\mathrm{C} 48-1$ is too strong that deleting $\operatorname{asp} A$ is not enough to reduce the virulence. On the other hand, chickens are usually infected with $P$. multocida through the digestive and respiratory tract in the wild. An intramuscular route of infection may not reveal the role of $\operatorname{asp} A$ in virulence compared to an experiment where chickens are inoculated with $P$. multocida in a manner that reflects natural transmission such as through the mouth, nose, or conjunctiva. Moreover, $A$. pleuropneumoniae and C. jejuni both grow in an anaerobic environment in the host while $P$. multocida does not. The survival pressure of anaerobic environment might be higher than that of iron limiting environment. Therefore, whether $\operatorname{asp} A$ could play a vital virulence factor only in anaerobic bacteria should be further studied.

\section{Conclusions}

In conclusion, this study demonstrated that $P$. multocida aspA was required for bacterial growth in complex medium and under anaerobic, acid, and iron-limited conditions. This study provides the first evidence for the role of $\operatorname{asp} A$ in iron acquisition. In addition, although the competitive index assay showed the wild-type strain outcompetes the aspA mutant strain, there was no significant difference in virulence between the mutant and the wild strains. The reasons need to be studied further.

\section{Materials and methods}

\section{Bacterial strains and growth conditions}

The wild-type bacterial strain used in these studies was P. multocida (C48-1), which was originally obtained from the China Veterinary Culture Collection Center. C48-1 is considered to be highly virulent in chickens. The bacterial strains and plasmids are described in Table 1. The $\operatorname{asp} A$ mutant $\triangle a s p A:: k a n$, was derived from the wild-type strain C48-1. Unless otherwise stated, all cultures were maintained in tryptone soy broth (TSB; Difco Laboratories, Detroit, MI, USA) in the presence or absence of $150 \mu \mathrm{M} 2,2^{\prime}$-dipyridyl (DPD; Sigma, Santa 
Clara, CA, USA), and in the presence or absence of oxygen at $37^{\circ} \mathrm{C}$. The following antibiotics were added to the selection media as required: kanamycin, $100 \mathrm{mg} / \mathrm{mL}$; chloramphenicol, $100 \mathrm{mg} / \mathrm{mL}$; ampicillin, $100 \mathrm{mg} / \mathrm{mL}$; spectinomycin, $100 \mathrm{mg} / \mathrm{mL}$ and gentamicin, $100 \mathrm{mg} / \mathrm{mL}$.

\section{Generation of the $\triangle a s p A:$ kan mutant and complemented strains}

The whole genome of $P$. multocida (C48-1) has not yet been sequenced, and all the primers were therefore based on the genomic sequence of Pm70 (GenBank accession: AE004439.1) (Table 2). AspA gene was deleted by allelic exchange through a recombinant suicide vector, which replaced the whole aspA gene with a $902 \mathrm{bp}$ kanamycinresistance cassette. Briefly, the $453 \mathrm{bp}$ upstream and 447 bp downstream fragments of the $P$. multocida aspA gene were amplified using aspAU1-aspAU2 and aspAD1aspAD2 primer sets (Table 2), respectively. The upstream and downstream fragments were fused by overlap polymerase chain reaction (PCR) using aspAU1-aspAD2 primers. The purified aspA deletion fragment was then cloned into pBC-Tn903 [39] at the Kpn I and BamH I restriction sites using T4 DNA ligase (Takara Bio Inc., Tokyo, Japan) to obtain the plasmid pBC-aspA. The kanamycin resistance $\left(\mathrm{kan}^{\mathrm{R}}\right)$ cassette amplified from pET-28a with the primers Kan-F1 and Kan-F2 (Table 2) was then inserted into the Not I and $S b f$ I sites of pBC-aspA to generate the plasmid $\mathrm{pBC}$-aspA-kan. This plasmid was subsequently introduced into $P$. multocida $\mathrm{C}_{48-1}$ via electroporation to obtain a single crossover strain on TSB agar plates containing kanamycin and chloramphenicol. The second crossover strain was selected by chloramphenicol sensitivity and kanamycin resistance. Candidate mutant clones were confirmed by PCR screening using primers T1 and T2 (Table 2). The wild-type and deleted alleles could be differentiated on the basis of the size of the amplicon by agarose gel electrophoresis. The mutant strain was designated $\triangle a s p A:: k a n$.

For complementation of aspA mutants strains in $P$. multocida, the amplification of the promoter sequence (328 bp) and coding sequence (1419 bp) of aspA gene using two primers, CaspA1/2 (Table 2), was cloned into pAL99S [40] to obtain the plasmid pAL99S-aspA. Then, the recombinant plasmid and an empty vector were transformed into the aspA mutant strain via electroporation respectively. The two strains were selected on TSA containing kanamycin and spectinomycin and further confirmed by PCR and RT-PCR using primers M1/2 (outside the multiple cloning site of pAL99S) and qaspA1/2 (Table 2). The complementary strain was designated $\mathrm{C} a s p A:: k a n$ and the control strain was designated $\triangle a s p A:: k a n$ (pAL99S).

\section{Growth in complex media under aerobic conditions}

We compared the growth rates among C48-1, $\triangle$ aspA:: kan, C $\triangle a s p A:: k a n$ and $\triangle a s p A:: k a n$ (pAL99S) by determining the growth curves of the three strains in different media. Overnight cultures in TSB were centrifuged at $2300 \mathrm{~g}$ for $5 \mathrm{~min}$ and diluted to optical density $\mathrm{OD}_{600}=1$ before subculture at 1:100 in $5 \mathrm{ml} \mathrm{TSB}, \mathrm{LB}$, and $\mathrm{MH}$ media, respectively, and incubation at $37{ }^{\circ} \mathrm{C}$ with constant shaking under aerobic conditions. Samples were collected every hour for $18 \mathrm{~h}$ to determine the $\mathrm{OD}_{600}$ and create a growth curve.

We further explored the amino acid catabolism function of $\operatorname{asp} A$ by comparing the growth abilities of the parent and mutant strains in $\mathrm{LB}$ and $\mathrm{MH}$ in the presence or absence of fumarate. Overnight cultures in TSB were centrifuged and diluted to $\mathrm{OD}_{600}=1$ before subculture at 1:100 in $5 \mathrm{ml} \mathrm{LB}$ and $\mathrm{MH}$ media in the presence or absence of $20 \mathrm{mM}$ fumarate (Sigma), respectively, followed by incubation at $37^{\circ} \mathrm{C}$ with constant shaking

Table 1 Strains and plasmids used in this study

\begin{tabular}{|c|c|c|}
\hline Strains or plasmids & Description & Source or reference \\
\hline \multicolumn{3}{|l|}{ Strains } \\
\hline C48-1 & Avian P. multocida C48-1. Capsulated and virulent & China Veterinary Culture Collection Center \\
\hline$\triangle a s p A:: k a n$ & aspA mutant strain of Avian P. multocida C48-1 & This work \\
\hline$C \triangle a s p A:: k a n$ & Complemented strain of $\triangle a s p A:: k a n$ & This work \\
\hline$\triangle a s p A:: k a n(p A L 99 S)$ & aspA mutant strain harboring an empty vector & This work \\
\hline \multicolumn{3}{|l|}{ Plasmids } \\
\hline pBC-Tn903 & Suicide vector, $\mathrm{Cm}^{\mathrm{R}}, \mathrm{Kan}^{\mathrm{R}}$ & {$[39]$} \\
\hline pBC-aspA & Containing left and right arms of aspA, $\mathrm{Cm}^{\mathrm{R}}$ & This work \\
\hline pBC-aspA-kan & Constructing aspA gene deletion mutants, $\mathrm{Cm}^{\mathrm{R}}, \mathrm{Kan}^{\mathrm{R}}$ & This work \\
\hline pAL99S & P. multocida expression plasmid, derivative of pAL99, Spec ${ }^{R}$ & {$[40]$} \\
\hline pAL99S-aspA & Containing the intact aspA, Spec $^{R}$ & This work \\
\hline pET-28a & Amplifing the kanamycin resistance cassette, $\operatorname{Kan}^{R}$ & Our Lab \\
\hline
\end{tabular}

$\mathrm{Kan}^{R}$ kanamycin resistance, $\mathrm{Cm}^{R}$ chloramphenicol resistance, $\operatorname{Spec}^{R}$ spectinomycin resistance 
Table 2 Primers used in this study

\begin{tabular}{|c|c|c|}
\hline Primer & Sequence $5^{\prime}-3^{\prime}$ & RE \\
\hline aspAU1 & CGGGGTACCCCCTAATGCAGAAGTAATTAA & Kpnl \\
\hline aspAU2 & CCTGCAGGATGCGGCCGCGCATTICGAGTGATGAACAAGT & Pstl/Notl \\
\hline aspAD1 & CGCGGCCGCATCCTGCAGGATAACTGTTAATTAACCCGCA & Notl/Pstl \\
\hline aspAD2 & CGCGAGCTCAGCGTGTAAGCAATATTTAG & Sacl \\
\hline Kan-F1 & ATAAGAATGCGGCCGCTCAGTGGAACGAAAACTC & Notl \\
\hline Kan-F2 & TGCACCTGCAGGTTAGAAAAACTCATCGAGCATC & Pstl \\
\hline aspA1 & ATGACAGTAACAAGAAAAGAAGT & 一 \\
\hline aspA2 & TTATTTATTCAACTTCGCTTTATAG & 一 \\
\hline $\mathrm{T} 1$ & TTCGGCATTTAGCAAACTGACGACG & 一 \\
\hline $\mathrm{T} 2$ & GGTGTCACACTTCCGTGCGTTAGAG & 一 \\
\hline CaspA1 & CGCGGATCCTITAATGATACAAGGGCTATGCTCA & $\mathrm{BamHI}$ \\
\hline CaspA2 & GGCGTCGACTTATTTATTCAACTTCGCTTTATAGG & Sal I \\
\hline M1 & GAAGAGTGCAGTTGGCTTGCG & 一 \\
\hline M2 & AAATCGCGAGGAATACTGACG & 一 \\
\hline qaspA1 & TTGTGGGGCGTATGTGATGG & 一 \\
\hline qaspA2 & ACTGGGTTGACTITGCTGGC & 一 \\
\hline q16s rRNA1 & TCACCGCAACATTCTGATTT & 一 \\
\hline q16s rRNA2 & CATACAGAGGGCAGCGAGA & 一 \\
\hline
\end{tabular}

Restriction endonuclease (RE) cleavage sites introduced into primers are underlined

under aerobic conditions. Samples were collected every hour for $18 \mathrm{~h}$ to determine the $\mathrm{OD}_{600}$ and create a growth curve. All growth experiments were performed twice independently with three replicates.

\section{Growth under anaerobic conditions}

AspA was shown to be essential for growth under anaerobic conditions in A. pleuropneumoniae [5] and $C$. jejuni [17]. To determine if aspA was also related to anaerobic tolerance in $P$. multocida, we compared the abilities of C48-1, $\triangle a s p A:: k a n, \mathrm{C}^{\triangle} a s p A:: k a n$ and $\triangle$ aspA::kan (pAL99S) to grow in TSB under anaerobic conditions. Briefly, overnight cultures in TSB were centrifuged and diluted to $\mathrm{OD}_{600}=1$ before subculture at 1:100 into $5 \mathrm{ml}$ TSB medium under aerobic or anaerobic conditions, in a MACS-MG-1000-controlled atmosphere workstation (DW Scientific, Japan), and then incubated at $37^{\circ} \mathrm{C}$ with constant shaking. The absorbance at $\mathrm{OD}_{600}$ was measured after $16 \mathrm{~h}$. All growth experiments were performed twice independently with three replicates.

Moreover, we explored the growth abilities of the parent and mutant strains in TSB under aerobic or anaerobic conditions in the presence or absence of fumarate. Overnight cultures were centrifuged and diluted to $\mathrm{OD}_{600}=1$ before subculture at 1:100 in $5 \mathrm{ml}$ TSB media under aerobic or anaerobic conditions in the presence or absence of $20 \mathrm{mM}$ fumarate, respectively, followed by incubation at $37^{\circ} \mathrm{C}$ with constant shaking. The absorbance at $\mathrm{OD}_{600}$ was measured after $16 \mathrm{~h}$. All growth experiments were performed twice independently with three replicates.

\section{Growth under acid conditions}

Overnight cultures in commercial TSB medium $(\mathrm{pH}=$ 7.3) were centrifuged at $2300 \mathrm{~g}$ for $5 \mathrm{~min}$ and diluted to $\mathrm{OD}_{600}=1$. Then the diluted cultures were subcultured at $1: 100$ in $5 \mathrm{ml} \mathrm{TSB}, \mathrm{pH}=7.3$ or TSB, $\mathrm{pH}=5.0$ and then incubated at $37^{\circ} \mathrm{C}$ with constant shaking for $1 \mathrm{~h}$ under aerobic conditions. The number of residual bacteria was counted by spreading serial dilutions onto TSA. All experiments were performed twice independently with three replicates.

\section{DPD-sensitivity assays}

We explored the effects of DPD concentrations on the growth of wild-type and mutant $P$. multocida, respectively $C 48-1$ and $\triangle a s p A:: k a n$ by examining their ironlimited growth in TSB containing the iron chelator DPD at $0,100,150,200,250$, and $300 \mu \mathrm{M}$. Briefly, overnight cultures in TSB were centrifuged at $2300 \mathrm{~g}$ for $5 \mathrm{~min}$ and diluted to $\mathrm{OD}_{600}=1$ before subculture at 1:100 into $\mathrm{ml}$ TSB medium containing $0,100,150,200,250$, and $300 \mu \mathrm{M}$ DPD, followed by incubation at $37^{\circ} \mathrm{C}$ with constant shaking under aerobic conditions. The absorbance at $\mathrm{OD}_{600}$ was measured after $16 \mathrm{~h}$. All growth experiments were performed twice independently with three replicates. 


\section{Growth under iron-depleted conditions}

We investigated the role of asp $A$ in iron acquisition by comparing the ability of C48-1, $\triangle$ aspA::kan, $\mathrm{C} \triangle a s p A::$ $k a n$ and $\triangle a s p A:: k a n$ (pAL99S) to grow in TSB in the presence or absence of DPD. Overnight cultures in TSB were centrifuged at $2300 \mathrm{~g}$ for $5 \mathrm{~min}$ and diluted to $\mathrm{OD}_{600}=1$ before subculture at $1: 100$ in $5 \mathrm{ml}$ TSB medium containing 0 or $150 \mu \mathrm{M}$ DPD, followed by incubation at $37^{\circ} \mathrm{C}$ with constant shaking under aerobic conditions. Samples were collected every hour for $18 \mathrm{~h}$ to measure the $\mathrm{OD}_{600}$ and create a growth curve. All growth experiments were performed twice independently with three replicates.

\section{Iron utilization under iron-depleted conditions}

We explored the effects of different iron ions, $\mathrm{FeCl}_{3}$ and $\mathrm{FeSO}_{4}$ (Takara), on the growth of $P$. multocida C48-1 and $\triangle a s p A:: k a n, C \triangle a s p A:: k a n$ and $\triangle a s p A:: k a n$ (pAL99S) under iron-depleted conditions. $\mathrm{FeCl}_{3}$ or $\mathrm{FeSO}_{4}$ was added to TSB at a final concentration of $100 \mu \mathrm{M}$, followed by overnight culture, centrifugation, and dilution to $\mathrm{OD}_{600}=1$ before subculture at $1: 100$ in $5 \mathrm{ml} \mathrm{TSB}$ containing 0 or $150 \mu \mathrm{M}$ DPD and incubation at $37^{\circ} \mathrm{C}$, with constant shaking in presence of oxygen. The absorbance at $\mathrm{OD}_{600}$ was measured after $16 \mathrm{~h}$. All growth experiments were performed twice independently with three replicates.

\section{Transcription of aspA under iron-depleted conditions}

C48-1, $\triangle$ aspA::kan and $\mathrm{C} \triangle a s p A:: k a n$ were grown in TSB at $37^{\circ} \mathrm{C}$ for $16 \mathrm{~h}$ with agitation. Overnight cultures in TSB were centrifuged at $2300 \mathrm{~g}$ for $5 \mathrm{~min}$ and diluted to $\mathrm{OD}_{600}=1$ before subculture at $1: 100$ in $5 \mathrm{ml} \mathrm{TSB}$ medium containing $0,100,150$, and $200 \mu \mathrm{M}$ DPD, followed by incubation at $37^{\circ} \mathrm{C}$ with constant shaking in presence of oxygen to reach $\mathrm{OD}_{600}=0.6$. The strains were collected by centrifugation and total RNA were extracted using an RNeasy Mini Kit (Qiagen, Hilden, Germany). All RNA isolated from samples was DNase treated with DNA-Free (Sigma) and reverse transcribed into cDNA using an iScript cDNA Synthesis Kit (Promega, Wisconsin, USA) and oligo (dT) primers before quantitative PCR (qPCR). The mRNA expression levels of $a s p A$ and the reference gene $16 s r R N A$ were quantified by real-time PCR using SYBR Green Master Mix (Roche Diagnostics, Shanghai, China). The primers used for real-time PCR were designed using Primer Premier 5 (Premier Biosoft, Palo Alto, CA, USA) (Table 2). Expression levels were normalized to $16 s \mathrm{r} R N A$ and presented as fold-change compared with the respective controls. All experiments were performed twice independently with three replicates.

\section{Biofilm-formation assay}

Biofilm formation by $\mathrm{C} 48-1, \triangle a s p A:: k a n$ and $\mathrm{C}^{\triangle}$ asp $A:$ : kan was assessed by crystal violet staining in 6-well plates (Sigma) as described previously [41]. Briefly, overnight cultures in TSB were centrifuged and diluted to contain approximately $10^{6}$ colony-forming units (CFU)/ $\mathrm{ml}$, and $200 \mu \mathrm{l}$ of the standardized inoculum was inoculated in TSB with or without $150 \mu \mathrm{M}$ DPD into triplicate wells in six-well plates. Uninoculated medium was used as a control. After incubation at $37^{\circ} \mathrm{C}$ for $48 \mathrm{~h}$ under aerobic conditions, the final culture density was determined by measuring the $\mathrm{OD}_{600}$. The cells were then stained with crystal violet, washed, and the crystal violet was solubilized with $95 \%$ ethanol. The total biomass was quantified by measuring the $\mathrm{OD}_{600}$ of the dissolved crystal violet. To avoid any effects of different growth rates caused by DPD, the biomass of the biofilms was measured by crystal violet staining and expressed relative to the final culture density. All growth experiments were performed twice independently with three replicates.

\section{Assessment of virulence in vivo}

We investigated the role of aspA in virulence in chickens in vivo. Briefly, healthy, 55-day-old healthy chickens were purchased from a chicken farm (HuBei, PR China) and housed in cages under a $12 \mathrm{~h}$ light/dark cycle. Throughout the experiment, chickens were kept at 25$28^{\circ} \mathrm{C}$ and provided with food and water ad libitum. C48-1 and $\operatorname{asp} A$ mutant were grown in TSB at $37^{\circ} \mathrm{C}$ for $16 \mathrm{~h}$ in TSB with agitation under aerobic conditions. Overnight cultures were then centrifuged and diluted to contain approximately 10 and $100 \mathrm{CFU}$, respectively. The chickens were divided randomly into five groups of 10 each and injected intramuscularly with 10 or 100 CFU of the wildtype C48-1 or mutant strain $\triangle a s p A::$ kan. The negative control group was injected with phosphate-buffered saline (PBS). Chicken mortality was recorded daily for a period of 12 days after the challenge. The remaining chickens were killed humanely with an intravenous injection of sodium pentobarbital $(100 \mathrm{mg} /$ $\mathrm{kg}$ bodyweight) at the end of the study.

\section{In vivo competition assay}

C48-1, aspA mutant and complemented strains were grown in TSB at $37^{\circ} \mathrm{C}$ for $16 \mathrm{~h}$ with agitation. Overnight cultures were then centrifuged and diluted to contain approximately $10^{3} \mathrm{CFU} / \mathrm{ml}$, respectively. C48-1 were then mixed with $\triangle a s p A:: k a n$ or $C \triangle a s p A:: k a n$ at a $1: 1$ ratio and 55-day-old healthy chickens were injected intramuscularly with $200 \mu \mathrm{l}$ of the mixture containing 100 $\mathrm{CFU}$ of each strain. At $24 \mathrm{~h}$ and $72 \mathrm{~h}$ after infection, tissue samples $(0.25 \mathrm{~g})$ from spleens and livers of the chickens (five per group) were collected, weighed, triturated in $900 \mu \mathrm{l}$ of PBS, and homogenized. Subsequently, the 
homogenates were 10 -fold serially diluted, and $100 \mu \mathrm{l}$ of the diluted suspensions were plated on TSA agar with or without kanamycin $(100 \mu \mathrm{g} / \mathrm{ml})$. The mutant (or complement) titer was calculated from the CFU recovered on TSA agar containing kanamycin, and the bacterial load of wild-type was obtained from the CFU recovered on TSA agar and subtracted the number of the mutant. The results were shown as the $\log 10$ competitive index.

\section{Statistical analysis}

All statistical analyses were performed using GraphPad Prism 5 (GraphPad Software Inc., San Diego, CA, USA) software for Windows. Differences were evaluated using Student's $t$-tests. The value of $P<0.05$ was considered significant.

\section{Abbreviations}

AspA: Aspartate ammonia-lyase; P. multocida: Pasteurella multocida; LB: LuriaBertani; MH: Mueller-Hinton; C. jejuni: Campylobacter jejuni; A. pleuropneumoniae: Actinobacillus pleuropneumoniae; TSB: Tryptone Soya Broth; TSA: Tryptic Soy Agar; Kan ${ }^{\mathrm{R}}$ : Kanamycin-resistance; CFU: Colony Forming Unit; DPD: 2,2'-dipyridyl

\section{Acknowledgements}

We would like to thank Prof. Dr. Qingping Luo for her careful review of the manuscript.

\section{Authors' contributions}

Experiments were conceived and designed by QL2 and HS. XW, LL1 and QL1 contributed to analysis and revision of the manuscript. Experiments were performed by ZW, LL2, and PL. The data were analyzed by CW. The manuscript was written by ZW. All authors read and approved the final manuscript.

\section{Funding}

This study was funded by the National Key Research and Development Program of China (2018YFD0500503), the National Natural Science Foundation of China (31873017) and the Technical System of National Broiler Industry (CARS-41-G13).

\section{Availability of data and materials}

The datasets generated and analysed during the current study are available from the corresponding author on reasonable request.

\section{Ethics approval and consent to participate}

All animal experimental procedures were sanctioned by the National Ethical Commission (Hubei Province P.R. China). All animal procedures were performed according to the regulations and guidelines established by the local ethic committee of the Hubei Academy of Agricultural Sciences (Hubei Province, P.R. China) and international standards for animal welfare.

\section{Consent for publication}

Not applicable.

\section{Competing interests}

The authors declare that they have no competing interests.

\section{Author details}

${ }^{1}$ Institute of Animal Husbandry and Veterinary Sciences, Hubei Academy of Agricultural Sciences, Special one, Nanhuyaoyuan, Hongshan District, Wuhan 430064, China. ${ }^{2}$ Animal Disease Prevention and Control Center of Yichang, Yichang 443000, China. ${ }^{3}$ Key Laboratory of Prevention and Control Agents for Animal Bacteriosis, Special 1, Nanhuyaoyuan, Hongshan District, Wuhan 430064, China.
Received: 27 June 2020 Accepted: 23 November 2020

Published online: 03 December 2020

\section{References}

1. Harper M, Boyce JD, Adler B. Pasteurella multocida pathogenesis: 125 years after Pasteur. FEMS Microbiol Lett. 2006;265(1):1-10.

2. Wilkie IW, Harper M, Boyce JD, Adler B. Pasteurella multocida: diseases and pathogenesis. Curr Top Microbiol Immunol. 2012;361:1-22.

3. Tang Q, Li W, Dai N, Gao Y, Han Y, Cheng G, Gu C. The role of Necroptosis, apoptosis, and inflammation in fowl cholera-associated liver injury in a chicken model. Avian Dis. 2017:61(4):491-502.

4. Nogrady N, Imre A, Rychlik I, Barrow PA, Nagy B. Genes responsible for anaerobic fumarate and arginine metabolism are involved in growth suppression in salmonella enterica serovar Typhimurium in vitro, without influencing colonisation inhibition in the chicken in vivo. Vet Microbiol. 2003:97(3-4):191-9.

5. Jacobsen I, Hennig-Pauka I, Baltes N, Trost M, Gerlach GF. Enzymes involved in anaerobic respiration appear to play a role in Actinobacillus pleuropneumoniae virulence. Infect Immun. 2005;73(1):226-34.

6. Spencer ME, Lebeter VM, Guest JR. Location of the Aspartase gene (aspA) on the linkage map of Escherichia coli K12. J Gen Microbiol. 1976;97(1):7382.

7. Falzone CJ, Karsten WE, Conley JD, Viola RE. L-aspartase from Escherichia coli: substrate specificity and role of divalent metal ions. Biochemistry. 1988; 27(26):9089-93.

8. van der Werf MJ, van den Tweel WJ, Kamphuis J, Hartmans S, de Bont JA. The potential of lyases for the industrial production of optically active compounds. Trends Biotechnol. 1994;12(3):95-103.

9. Hu Y, Lu P, Zhang Y, Li L, Chen S. Characterization of an aspartatedependent acid survival system in Yersinia pseudotuberculosis. FEBS Lett. 2010:584(11):2311-4

10. Van Hellemond JJ, Tielens AG. Expression and functional properties of fumarate reductase. Biochem J. 1994:304(Pt 2):321-31.

11. Holmes K, Mulholland F, Pearson BM, Pin C, McNicholl-Kennedy J, Ketley JM, Wells JM. Campylobacter jejuni gene expression in response to iron limitation and the role of Fur. Microbiology. 2005;151(Pt 1):243-57.

12. Deslandes V, Nash JH, Harel J, Coulton JW, Jacques M. Transcriptional profiling of Actinobacillus pleuropneumoniae under iron-restricted conditions. BMC Genomics. 2007:8:72

13. Dumpala PR, Peterson BC, Lawrence ML, Karsi A. Identification of differentially abundant proteins of Edwardsiella ictaluri during Iron restriction. PLoS One. 2015;10(7):e0132504

14. Luo Q, Kong L, Dong J, Zhang T, Wang H, Zhang R, Lu Q, Chen H, Shao H, Jin M. Protection of chickens against fowl cholera by supernatant proteins of Pasteurella multocida cultured in an iron-restricted medium. Avian Pathol. 2019:48(3):221-9.

15. Litwin CM, Calderwood SB. Role of iron in regulation of virulence genes. Clin Microbiol Rev. 1993:6(2):137-49.

16. Guo J, Man Z, Rao Z, Xu M, Yang T, Zhang X, Xu Z. Improvement of the ammonia assimilation for enhancing $L$-arginine production of Corynebacterium crenatum. J Ind Microbiol Biotechnol. 2017;44(3):443-51.

17. Guccione E, Leon-Kempis Mdel R, Pearson BM, Hitchin E, Mulholland F, van Diemen PM, Stevens MP, Kelly DJ. Amino acid-dependent growth of campylobacter jejuni: key roles for aspartase (AspA) under microaerobic and oxygen-limited conditions and identification of AspB (Cj0762), essential for growth on glutamate. Mol Microbiol. 2008;69(1):77-93.

18. Alteri CJ, HimpsI SD, Engstrom MD, Mobley HL. Anaerobic respiration using a complete oxidative TCA cycle drives multicellular swarming in Proteus mirabilis. mBio. 2012;3(6):e00365-12. https://doi.org/10.1128/mBio.00365-12.

19. Rastogi VK, Watson RJ. Aspartate aminotransferase activity is required for aspartate catabolism and symbiotic nitrogen fixation in rhizobium meliloti. J Bacteriol. 1991;173(9):2879-87.

20. Soellner S, Rahnert M, Siemann-Herzberg M, Takors R, Altenbuchner J. Evolution of pyruvate kinase-deficient Escherichia coli mutants enables glycerol-based cell growth and succinate production. J Appl Microbiol. 2013;115(6):1368-78

21. Takahashi N, Sato T, Yamada T. Metabolic pathways for cytotoxic end product formation from glutamate- and aspartate-containing peptides by Porphyromonas gingivalis. J Bacteriol. 2000;182(17): 4704-10. 
22. Wang M, Zhang P, Zhu D, Wang M, Jia R, Chen S, Sun K, Yang Q, Wu Y, Chen X, et al. Identification of the ferric iron utilization gene B739_1208 and its role in the virulence of R. anatipestifer CH-1. Vet Microbiol. 2017;201:162-9.

23. Jatuponwiphat $\mathrm{T}$, Chumnanpuen $\mathrm{P}$, Othman $\mathrm{S}$, Teerasak E, Vongsangnak W. Iron-associated protein interaction networks reveal the key functional modules related to survival and virulence of Pasteurella multocida. Microb Pathog. 2019;127:257-66.

24. Woods SA, Miles JS, Roberts RE, Guest JR. Structural and functional relationships between fumarase and aspartase. Nucleotide sequences of the fumarase (fumC) and aspartase (aspA) genes of Escherichia coli K12. Biochem J. 1986;237(2):547-57.

25. Mey AR, Wyckoff EE, Kanukurthy V, Fisher CR, Payne SM. Iron and fur regulation in Vibrio cholerae and the role of fur in virulence. Infect Immun. 2005;73(12):8167-78.

26. Sebastian S, Agarwal S, Murphy JR, Genco CA. The gonococcal fur regulon: identification of additional genes involved in major catabolic, recombination, and secretory pathways. J Bacteriol. 2002;184(14):3965-74

27. Kim YC, Miller CD, Anderson AJ. Transcriptional regulation by iron of genes encoding iron- and manganese-superoxide dismutases from Pseudomonas putida. Gene. 1999;239(1):129-35.

28. Park SJ, Gunsalus RP. Oxygen, iron, carbon, and superoxide control of the fumarase fumA and fumC genes of Escherichia coli: role of the arcA, fnr, and soxR gene products. J Bacteriol. 1995;177(21): 6255-62.

29. Philips J, Van den Driessche N, De Paepe K, Prevoteau A, Gralnick JA, Arends JBA, Rabaey K. A Novel Shewanella Isolate Enhances Corrosion by Using Metallic Iron as the Electron Donor with Fumarate as the Electron Acceptor. Appl Environ Microbiol. 2018;84:1-19. https://doi.org/10.1128/AEM.01154-18.

30. Dong Y, Geng J, Liu J, Pang M, Awan F, Lu C, Liu Y. Roles of three TonB systems in the iron utilization and virulence of the Aeromonas hydrophila Chinese epidemic strain NJ-35. Appl Microbiol Biotechnol. 2019;103(10): 4203-15.

31. Rizzi A, Roy S, Bellenger JP, Beauregard PB. Iron Homeostasis in Bacillus subtilis Requires Siderophore Production and Biofilm Formation. Appl Environ Microbiol. 2019;85(3):e02439-18. https://doi.org/10.1128/AEM.0243918.

32. Swarupa V, Chaudhury A, Sarma P. Iron enhances the peptidyl deformylase activity and biofilm formation in Staphylococcus aureus. 3 Biotech. 2018;8(1): 32.

33. Yu S, Ma L. Iron uptake and biofilm formation in Pseudomonas aeruginosa. Sheng Wu Gong Cheng Xue Bao. 2017;33(9):1489-512.

34. Liu Q, Liu B, Li W, Zhao X, Zuo W, Xing D. Impact of ferrous Iron on microbial Community of the Biofilm in microbial fuel cells. Front Microbiol. 2017;8:920.

35. Oh $\mathrm{E}$, Andrews $\mathrm{K}$, Jeon $\mathrm{B}$. Enhanced biofilm formation by ferrous and ferric Iron through oxidative stress in campylobacter jejuni. Front Microbiol. 2018; 9:1204.

36. Kang D, Kirienko NV. Interdependence between iron acquisition and biofilm formation in Pseudomonas aeruginosa. J Microbiol. 2018; 56(7):449-57.

37. Lu F, Miao S, Tu J, Ni X, Xing L, Yu H, Pan L, Hu Q. The role of TonBdependent receptor TbdR1 in Riemerella anatipestifer in iron acquisition and virulence. Vet Microbiol. 2013;167(3-4):713-8.

38. Porcheron G, Dozois CM. Interplay between iron homeostasis and virulence: Fur and RyhB as major regulators of bacterial pathogenicity. Vet Microbiol. 2015;179(1-2):2-14.

39. Guo D, Lu Y, Liu J, Yuan D, Zhang A, Jiang Q, Lin H, Si C, Qu L. Construction and characterization of aroA deletion mutant of Pasteurella multocida strain C51-17. Wei Sheng Wu Xue Bao. 2012;52(4):526-31.

40. Harper M, St Michael F, John M, Vinogradov E, Steen JA, van Dorsten L, Steen JA, Turni C, Blackall PJ, Adler B, et al. Pasteurella multocida Heddleston serovar 3 and 4 strains share a common lipopolysaccharide biosynthesis locus but display both inter- and intrastrain lipopolysaccharide heterogeneity. J Bacteriol. 2013;195(21):4854-64.

41. Garcia CA, Alcaraz ES, Franco MA, Passerini de Rossi BN. Iron is a signal for Stenotrophomonas maltophilia biofilm formation, oxidative stress response, OMPs expression, and virulence. Front Microbiol. 2015:6:926.

\section{Publisher's Note}

Springer Nature remains neutral with regard to jurisdictional claims in published maps and institutional affiliations.

\section{Ready to submit your research? Choose BMC and benefit from:}

- fast, convenient online submission

- thorough peer review by experienced researchers in your field

- rapid publication on acceptance

- support for research data, including large and complex data types

- gold Open Access which fosters wider collaboration and increased citations

- maximum visibility for your research: over $100 \mathrm{M}$ website views per year

At $\mathrm{BMC}$, research is always in progress.

Learn more biomedcentral.com/submissions 